\title{
Network Pharmacology-Based Strategy for Predicting Therapy Targets of Tripterygium wilfordii on Acute Myeloid Leukemia
}

\section{Tingting Fang}

The Affiliated Hospital of Southwest Medical University

\section{Lanqin Liu}

The Affiliated Hospital of Southwest Medical University

wenjun liu ( $\square$ liuwenjun_l@163.com)

The Affiliated Hospital of Southwest medical university https://orcid.org/0000-0002-7205-0598

\section{Research}

Keywords: Tripterygium wilfordii, network pharmacology, active compounds, anti-AML

Posted Date: May 21st, 2020

DOI: https://doi.org/10.21203/rs.3.rs-29959/v1

License: (1) (1) This work is licensed under a Creative Commons Attribution 4.0 International License.

Read Full License

Version of Record: A version of this preprint was published at Medicine on December 11th, 2020. See the published version at https://doi.org/10.1097/MD.0000000000023546. 


\section{Abstract}

Background. Acute myeloid leukemia (AML) is a common malignant tumor of the hematopoietic system. How to extend the survival time of AML patients and improve their prognosis is still a major medical problem. Chinese medicine has a long history in treating AML. Tripterygium wilfordii (TW) is a traditional Chinese medicine. With the deepening of pharmacological research of traditional Chinese medicine, triptolide, one of its active ingredients, has been proven to have a positive effect on the treatment of AML. Therefore冈this study aimed on studying the potential therapeutic targets and pharmacological mechanism of TW in Acute myeloid leukemia (AML) based on network pharmacology.

Methods. The active components of TW were obtained by network pharmacology through oral bioavailability, drug-likeness filtration. Comparative analysis was used to study the overlapping genes between active ingredient's targets and AML treatment-related targets. Using STRING database to analyze interactions between overlapping genes. KEGG pathway analysis and Gene Ontology enrichment analysis were conducted in DAVID. These genes were analyzed for survival in OncoLnc database.

Key findings. We screened 53 active ingredients, the results of comparative analysis showed that 8 active ingredients had an effect on AML treatment. Based on the active ingredients and overlapping genes, we constructed the Drug-Compounds-Genes-Disease Network. Survival analysis of overlapping genes indicated that some targets possess a significant influence on patients' survival and prognosis. The enrichment analysis showed that the main pathways of targets are Toll-like receptor signaling pathway, NF-kappa B signaling pathway and HIF-1 signaling pathway.

Conclusion. This study, using a network pharmacologic approach, provides another strategy that can help us to understand the mechanisms by which TW treats AML comprehensively.

\section{Introduction}

Acute myeloid leukemia (AML) is one of the common tumors in the blood system and is caused by the cloning and amplification of undifferentiated myeloid progenitor cells ${ }^{(1,2)}$. Impaired hematopoietic function and bone marrow failure are the most important features of AML, which can lead to death ${ }^{(3)}$. Although advances in AML treatment have significantly improved the prognosis of young patients, a mass of the new cases are elderly, and the prognosis of the elderly is still very poor ${ }^{(4)}$. Even with current treatments, the prognosis is poor, with a five-year relative survival rate of only $30-50 \%{ }^{(5)}$.

Traditional Chinese medicine (TCM) doctors in China have been using TCM clinically for more than 2,500 years $^{(6)}$. Some traditional Chinese medicines have been proven to have a therapeutic effect on $A M L$, such as arsenic trioxide ${ }^{(7)}$. And some other experiments also prove that traditional Chinese medicine has a

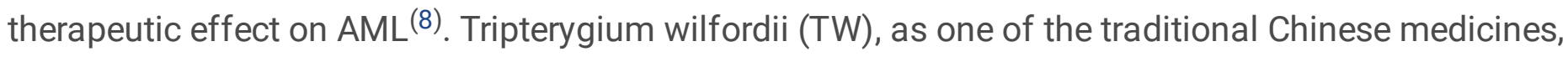
there is evidence to show that the traditional Chinese medicines have anti-tumor ability ${ }^{(9)}$. Proved by research, through mitochondrial-mediated pathway, triptolide can cause apoptosis of acute myeloid 
leukemia cells ${ }^{(10)}$. Although the active ingredients in TW have been identified, the mechanism by which the active ingredients achieve anti-AML pharmacological effects remains to be studied. In recent years, not only the continuous development of system biology, but also network pharmacology and computer technology are booming. In addition, the Internet pharmacology has played an important role in many fields, such as drug identification, active ingredient discovery, mechanism of action research, and drug efficacy and safety evaluation in preclinical research ${ }^{(11)}$. So as a helpful tool, it could assist us to further understand what the drugs can do, and help us to deepen our insights on how to improve the discovery of drugs in diseases $(12,13)$.

In this study, the potential mechanism of TW treatment AML was analyzed by the method of network pharmacology. The target protein of TW active ingredient and the target of AML treatment were intersected. With TW and AML as the center, Drug-Compounds-Genes-Disease Network was constructed. Construct a protein-protein interaction (PPI) network based on overlapping genes, and conduct enrichment analysis of GO and KEGG pathways. We hope to explore TW's potential therapeutic targets in anti-AML, and provide a basis for the study of TW pharmacological mechanism (Figure 1).

\section{Method}

\subsection{Screening of Active Compounds and Target Protein}

The active compounds of Tripterygium wilfordii and their target proteins were obtained from the Traditional Chinese Medicine Systems Pharmacology Database and Analysis Platform (TCMSP, http://tcmspw.com). As a special database which contains a lot of herbs, active constituents and their targets $\triangle T C M S P$ is established by using the pharmacology of traditional Chinese medicine systems ${ }^{(14)}$. It also include some pharmacokinetic properties of active compounds, such as oral bioavailability (OB), drug-likeness (DL), and others. $\mathrm{OB}$, as one of the most crucial pharmacokinetic parameters, it indicates the ratio of the drug taken to the blood circulation ${ }^{(15)}$. The larger the $\mathrm{OB}$ value of compounds, the more likely it is to become a clinical drug. The similarity between a compound and a drug which is already known can be expressed in terms of $\mathrm{DL}^{(16)}$. It represents that although the compounds is not a drug, it has the possibility of becoming a drug.

So among these pharmacokinetic properties, $\mathrm{OB}$ and $\mathrm{DL}$ are considered to be the most important evaluation indicators of whether they can become drugs $(17,18)$. Due to the poor pharmacological activity of most compounds in traditional Chinese medicine, they cannot effectively act on the target protein. Therefore, $\mathrm{OB} \geq 30 \%$ and $\mathrm{DL} \geq 0.18$, which are considered as indicators for screening a clinical drug ${ }^{(19}$, 20). In our study, we also used those indicators as our screening criteria.

\subsection{The Acquisition of Gene Targets for Acute Myeloid Leukemia}


The target genes for Acute Myeloid Leukemia in this study were collected from two database. The first database is GeneCards (https://www.genecards.org/, version 4.9.0), as a database which contains a lot of genes, such as genome, transcriptome, proteome and genetics, as well as clinical and functional information from 150 web sources ${ }^{(21)}$. The other database is the Online Mendelian Inheritance in Man(OMIM) database(http://www.omim.org/) ${ }^{(22)}$.

\subsection{Drug-Compounds-Genes-Disease (D-C-G-D) Network Construction}

At the beginning, we took the target genes of Tripterygium wilfordii's active component and the therapeutic targets for AML obtained from the above two databases to get the overlapping genes. After that, we integrated the information of the component, drug,genetic, diseases. Then we used Cytoscape software (v.3.6.1,https://cytoscape.org/) to visually analyze this information to construct D-C-G-D (23, 24).

\subsection{PPI networks construction}

Protein-protein interaction data were gotten from the STRING database (https://string-db.org/, version $11.0)$, which is a database that can predict known protein interactions ${ }^{(25)}$.

\subsection{Survival Analysis}

The information for these gene survival analyses comes from the OncoLnc database (http://www.oncolnc.org/). It contains RNA-SEQ expression and survival data of 8,647 patients' mRNAs and miRNAs in 21 cancer studies which are conducted by the Cancer Genome Atlas (TCGA) ${ }^{(26)}$.

\subsection{Gene Ontology Enrichment Analysis}

In this study, processed by DAVID6.8 (https://david.ncifcrf.gov/), every genes' GO as well as KEGG enrichment analysis were obtained. Three aspects are contained in $\mathrm{GO}$ enrichment analysis: biological process (BP), molecular function (MF), and cellular component $(\mathrm{CC})^{(27,28)}$. All the GO and KEGG enrichment analysis results were selected by $P$ value less than 0.05 as the critical criterion.

\section{Results}

\subsection{Screening of Active Compounds and Target}

To get the active compounds of TW, OB and DL which are two ADME parameters were used for screening the date which download from TCMSP. By using the value of $O B$ is bigger than 30 and the value of DL is 
bigger than 0.18 as the standard, we obtained 53 active compounds (Supplementary 1 ). Then we download the date about 53 active compounds' target. After deleting the useless items, 64 known targets were finally obtained. The details of these target were shown in Supplementary2.

\subsection{The Collection of Known Therapeutic targets for AML}

From Gene Card database, altogether 11619 Therapeutic targets which are already known for AML were acquired. In order to improve the accuracy of our data, Gifts greater than 10 were used as a standard to streamline the data. After that, 1093 Known therapeutic targets were collected. Then, we download 348 known therapeutic targets from OMIM to enrich our data.

\subsection{Screening the overlapping gene and Constructing D-C-G- D Network}

By taking the intersection of drug targets and disease targets, we got 37 genes (Figure $2 \mathrm{~A}$ ) and 8 active compounds (Table1), which means that these genes could play a major role in TW treatment of AML. In order to clarify the potential mechanism of TW's effect on AML, we constructed a D-C-G-D network by Cytoscape software (Figure 2 B).

Table 1

Showing the eight active compounds from Tripterygium wilfordii (TW) and their corresponding predicted oral bioavailability (OB), drug-likeliness (DL).

\begin{tabular}{|llll|}
\hline Mol ID & Molecule Name & $\begin{array}{c}\text { OB } \\
(\%)\end{array}$ & DL \\
\hline MOL000296 & hederagenin & 36.91 & 0.75 \\
\hline MOL003182 & $(+)$-Medioresinol di-O-beta-D-glucopyranoside_qt & 60.69 & 0.62 \\
\hline MOL003184 & $81827-74-9$ & 45.42 & 0.53 \\
\hline MOL003185 & $\begin{array}{l}\text { (1R,4aR,10aS)-5-hydroxy-1-(hydroxymethyl)-7-isopropyl-8-methoxy- } \\
\text { 1,4a-dimethyl-4,9,10,10a-tetrahydro-3H-phenanthren-2-one }\end{array}$ & 48.84 & 0.38 \\
\hline MOL003187 & triptolide & 51.29 & 0.68 \\
\hline MOL003196 & Tryptophenolide & 48.5 & 0.44 \\
\hline MOL003199 & 5,8-Dihydroxy-7-(4-hydroxy-5-methyl-coumarin-3)-coumarin & 61.85 & 0.54 \\
\hline MOL003217 & Isoxanthohumol & 56.81 & 0.39 \\
\hline
\end{tabular}

\subsection{Construction and Analysis of Target Proteins PPI Network}


For the purpose of figuring out how overlapping genes interact, we uploaded the information of them to the STRING database and then structure a PPI network, which contained 37 nodes and 324 edges (Figure $3 \mathrm{~A})$. And we show the bar plot the top 20 genes in the PPI network in Figure 3B. The result of PPI network indicate that there is a complex relationship between these genes.

\subsection{Survival Analysis}

To clarify the impact of these genes on the survival and prognosis of $A M L$, we performed survival analysis on them. And the results of $\mathrm{P}<0.05$ showed in Figure 4, the results indicated that some genes play an important role in the AML patients' survival and prognosis. The result meant that some active compounds in TW can act on these genes to improve the prognosis of patients.

\subsection{Gene Ontology Enrichment Analysis}

For the purpose of figuring out what the relationships between 37 genes and AML are, we carried out GO enrichment analysis on these genes in DAVID database and the result of GO enrichment analysis included three different levels: BP, MF, and CC. We selected the top 10 according to the $P$ value from small to large and shown in the figure5. The results indicated that TW treats AML through various biological processes, including reaction to drug(G0:0042493), negative regulation of apoptotic process(GO:0043066), reaction to cytokine(GO:0034097), and others. In the CC classification, nucleus is the main classification of the target proteins. The enrichment analysis results indicated that the active ingredients of TW have ability to affect the transcription of AML cell genes to treat AML.

\subsection{KEGG Enrichment Analysis}

To further explore how TW affects AML through these target genes, we uploaded these 37 genes to the DAVID database and performed KEGG enrichment analysis. And we selected the top 10 according to the $P$ value from small to large and shown in the figure6. KEGG pathway analysis of these genes presented several pathways correlative with the development and treatment of AML including Pathways in cancer(hsa05200), NF-kappa B signaling pathway(hsa04064), Transcriptional misregulation in cancer(hsa05202), HIF-1 signaling pathway(hsa04066), and others. We can find that a pathway includes multiple proteins, and a protein is also involved in multiple pathways. This complex regulatory relationship indicates that the active pharmacological components in TW may be used to treat some diseases including AML through these signaling pathways.

\section{Discussion}

Chinese medicine has a good effect and a long history for the treatment of various complex illness. In the research of active pharmaceutical ingredients, the method based on network interactions is of great help 
in deepening the understanding of the role of drugs in multi-layer information ${ }^{(29)}$. The interaction network of drugs, active ingredients, diseases and target proteins is an important way to express the mechanism of drugs and their active ingredients in the treatment of a certain disease, which helps to deepen the understanding of the therapeutic effects of drugs and offer a theoretical basis for new drugs development ${ }^{(30)}$. As an important pharmacological research method, network pharmacology can better demonstrate the relationship between drugs, components and targets. The application of this idea in the research of traditional Chinese medicine can help to understand the interaction mechanism of multicomponent multi-target model of traditional Chinese medicine ${ }^{(31)}$.

In this research, by taking the intersection of the active ingredient target of TW and the target of AML treatment, we obtained 37 target genes and eight active compounds. In these eight active compounds, Triptolide has been shown to have a therapeutic effect on $\operatorname{AML}{ }^{(9,32)}$. Triptolide can inhibit the expression of MDM2 and thereby inhibit the p53-dependent pathway to achieve the curative effect on $\mathrm{AML}^{\left({ }^{(3)}\right)}$. Other active ingredients in TW have also been shown to have therapeutic effects on other cancers. Such as Hederagenin, it can inhibit the proliferation of cervical cancer CaSki cells and promote their apoptosis by blocking the STAT3 pathway ${ }^{(34)}$. And Isoxanthohumol can inhibit the formation of lung metastasis in B16-F10 murine melanoma model ${ }^{(35)}$. Tripterygium also exhibited significant inhibitory effects on many cancer cells ${ }^{(36)}$.

On the other hand, it can be seen from the D-C-G-D Network that these active ingredients have a common target, which shows that they have some synergistic effects in the treatment of AML. As one of the predicted targets of TW for the treatment of AML, PTGS2 can be acted by 7 active compounds in TW. It has been reported that it can selectively inhibit cox-2 and play a role in the treatment of AML ${ }^{(36-38)}$. This means that multiple active ingredients in traditional Chinese medicine can act on a single target to take effect in the treatment of diseases. As for the active component, Triptolide is associated with 25 target genes, such as PTGS2, CD40, CASP3, JUN, etc., which is consistent with the fact that a single active compound can act on multiple targets.

In order to clarify the impact of these genes on the survival and prognosis of patients, we conducted a survival analysis of these 37 genes. The results show that some of these genes have a significant impact on the survival and prognosis of patients. And some genes have been experimentally proven to be the target of TW treatment of diseases such as AR, CD14, BCL2 ${ }^{(39-41)}$. This means that these genes may be the crucial target of TW in the treatment of leukemia.

Next, we conducted GO and KEGG enrichment analysis on these target genes. The major signaling pathways related to AML were found to include: Pathways in cancer, Toll-like receptor signaling pathway, NF-kappa B signaling pathway, Transcriptional misregulation in cancer, HIF-1 signaling pathway, and other pathways closely connected to the occurrence and development of AML. This indicates that Tripterygium wilfordii may have multiple active ingredients through multiple pathways to prevent and treat AML. Such as Toll-like receptor signaling pathway, as a part of the innate immune system, studies 
have shown that various hematological malignant cells, including AML cells, express TLRs, and TLR agonists may directiy effect on the leukemic cells ${ }^{(38)}$. Due to the indirect anti-leukemia effect caused by the activation / stimulation of normal immuneocompetent cells, such as eradicating residual leukemia cells after induction therapy, TLR is considered a possible drug target in AML ${ }^{(42)}$. In addition, the release of pro-inflammatory cytokines due to TLR stimulation can enhance the immunogenicity of AML blasts, making it easier to treat ${ }^{(43)}$. It is well known that in most patients with AML, NF-KB is constitutively activated, which helps to resist apoptosis. Studies have shown that inhibiting NF-KB can induce apoptosis in AML cells ${ }^{(44)}$. Studies have shown that HIF1a promotes the proliferation of AML cells through a pro-tumoral chemokine factor signaling pathway ${ }^{(45)}$.

Due to the complexity of traditional Chinese medicine ingredients and the limitations of experimental research methods, there are no reports on the effective ingredients, targets and mechanisms of TW for AML treatment. This study shows that using network pharmacology to study the effective ingredients and therapeutic targets of TW in the treatment of AML is of great significance for deepening the understanding of its related mechanism of action and discovering new effective ingredients. For the first time, the multi-targets and multi-pathways that play a role in AML are used to explain the molecular mechanism of Tripterygium wilfordii's therapeutic effect. However, the pathogenesis of AML is more complicated. Signaling pathways cross and regulate each other, presenting a pattern of multiple pathways and multiple targets. In-depth analysis of the anti-AML pharmacological actions of Tripterygium wilfordii, as well as the targets and pathways of the active ingredients are necessary to be further verified.

\section{Conclusion}

In this study, 8 active compounds with TW between AML and 37 overlapping genes were selected by the method of network pharmacology. After that, we constructed a PPI network of these genes, and conducted a survival analysis on the top 20 nodes to identify the effect of these genes on patients' survival and prognosis. The enrichment analysis of GO and KEGG was conducted to find the potential mechanism of action of TW in AML treatment. In conclusion, this study, using a network pharmacologic approach, provides another strategy to understand the mechanisms comprehensively by which TW treats AML.

\section{Abbreviations}

AML: Acute myeloid leukemia; TW: Tripterygium wilfordii; TCM: Traditional Chinese medicine; PPI: protein-protein interaction; OB: oral bioavailability; DL: drug-likeness; D-C-G-D: Drug-Compounds-GenesDisease; TCGA: the Cancer Genome Atlas;

\section{Declarations}




\section{Ethics approval and consent to participate}

Not applicable

\section{Consent for publication}

Not applicable

\section{Availability of data and materials}

All data in the manuscript are available and they are showed in figures, tables and supplement file.

\section{Competing interests}

The authors declare that they have no conflicts of interest concerning this work.

\section{Funding}

Basic Research Project of Sichuan Province, Grant/Award Number: 2019YJ0690; Major Science and Technology Projects in Sichuan Province, Grant/Award Number: 2019YFS0531

\section{Authors' contribution}

Wenjun Liu and Tingting Fang conceived and designed the studies. Lanqin Liu participated in this work. All authors participated in drafting of the manuscript and revising it before final submission.

\section{Acknowledgements}

This research work was supported by Basic Research Project of Sichuan Province, Grant/Award Number: 2019YJ0690; Major Science and Technology Projects in Sichuan Province, Grant/Award Number: 2019 YFS0531.

\section{References}

1. Schratz KE, Armanios M. Cancer and myeloid clonal evolution in the short telomere syndromes. Current Opinion in Genetics \& Development. 2020;60:112-8.

2. F. CS, L. LR. Genetic and epigenetic determinants of AML pathogenesis. Seminars in Hematology. 2018:S0037196318300970-. 
3. Weinberg OK, Sohani AR, Bhargava P, Nardi V. Diagnostic work-up of acute myeloid leukemia. American Journal of Hematology. 2017;92(3):317-21.

4. Shah A, Andersson ML, Rachet B, Bj?Rkholm M, Lambert PC. Survival and cure of acute myeloid leukaemia in England, 1971-2006: a population-based study. British Journal of Haematology. 2013;162(4):509-16.

5. Ravandi F, Burnett AK, Agura ED, Kantarjian HM. Progress in the treatment of acute myeloid leukemia. Cancer. 2007;110(9):1900-10.

6. Wang J, Wong YK, Liao F. What has traditional Chinese medicine delivered for modern medicine? Expert Reviews in Molecular Medicine. 2018;20.

7. Rui W, Ying L, Ping G, Janice G, Samuel W, Jing Y. Arsenic trioxide and sorafenib induce synthetic lethality of FLT3-ITD acute myeloid leukemia cells. Molecular Cancer Therapeutics. 2018:molcanther.0298.2017-.

8. Dang W, Wang W, Chen W, Lian F, Li L, Ying H, et al. Pharmacologic inhibition of dihydroorotate dehydrogenase induces apoptosis and differentiation in acute myeloid leukemia cells. Haematologica.haematol.2018.188185-.

9. Giri B, Gupta VK, Yaffe B, Modi S, Saluja A. Pre-clinical evaluation of Minnelide as a therapy for acute myeloid leukemia. Journal of Translational Medicine. 2019;17(1).

10. Carter BZ, Mak DH, Shi Y, Fidler JM, Chen R, Ling X, et al. MRx102, a triptolide derivative, has potent antileukemic activity in vitro and in a murine model of AML. Leukemia. 2012;26(3):443-50.

11. Ye H, Liu Q, Wei J. Construction of Drug Network Based on Side Effects and Its Application for Drug Repositioning. Plos One. 2014;9.

12. Zhang RZ, Yu SJ, Bai H, Ning K. TCM-Mesh: The database and analytical system for network pharmacology analysis for TCM preparations. Scientific reports. 2017;7(1):2821.

13. Chen L, Cao Y, Zhang H, Lv D, Zhao Y, Liu Y, et al. Network pharmacology-based strategy for predicting active ingredients and potential targets of Yangxinshi tablet for treating heart failure. Journal of Ethnopharmacology. 2018:S0378874117320901.

14. Ru J, Li P, Wang J, Zhou W, Li B, Huang C, et al. TCMSP: a database of systems pharmacology for drug discovery from herbal medicines. Journal of Cheminformatics. 2014;6(1):13.

15. Garg AX, Moist L, Pannu N, Tobe S, Walsh M, Weir M. Bioavailability of oral curcumin. Canadian Medical Association Journal. 2019;191(15):E428-E.

16. Hu Q, Feng M, Lai L, Pei J. Prediction of Drug-Likeness Using Deep Autoencoder Neural Networks. Frontiers in Genetics. 2018;9.

17. Ali R, Al-Mohizea AM, Al-Jenoobi FI, Alam MA. Understanding and Managing Oral Bioavailability: Physiological Concepts and Patents. Recent Patents on Anti Cancer Drug Discovery. 2015;10(1).

18. Tao W, Xue X, Xia W, Li B, Wang Y, Yan L, et al. Network pharmacology-based prediction of the active ingredients and potential targets of Chinese herbal Radix Curcumae formula for application to cardiovascular disease. Journal of Ethnopharmacology. 2013;145(1):1--10. 
19. Wenjie, Song, Shenglou, Ni, Yanling, Fu, et al. Uncovering the mechanism of Maxing Ganshi Decoction on asthma from a systematic perspective: A network pharmacology study. Scientific reports. 2018.

20. Yang L, Liu W, Hu Z, Yang M, Li J, Fan X, et al. A Systems Pharmacology Approach for Identifying the Multiple Mechanisms of Action of the Wei Pi Xiao Decoction for the Treatment of Gastric Precancerous Lesions. Evidence-based Complementary and Alternative Medicine. 2019;2019:1-15.

21. Nishimura D. GeneCards. Encyclopedia of Genetics Genomics Proteomics \& Informatics. 2001;2(2).

22. Ada H, Scott AF, Amberger JS, Bocchini CA, A. MV. Online Mendelian Inheritance in Man (OMIM), a knowledgebase of human genes and genetic disorders. Nucleic Acids Research. 2005(suppl_1):suppl_1.

23. Su G, Morris JH, Demchak B, Bader GD. Biological Network Exploration with Cytoscape 3. Curr Protoc Bioinformatics. 2014;47(1).

24. Doncheva NT, Morris JH, Gorodkin J, Jensen LJ. Cytoscape StringApp: Network Analysis and Visualization of Proteomics Data. Journal of proteome research. 2019;18(2):623-32.

25. Damian S, Andrea F, Stefan W, Kristoffer F, Davide H, Jaime HC, et al. STRING v10: protein-protein interaction networks, integrated over the tree of life. Nucleic Acids Research. 2014(D1):D1.

26. Anaya J. OncoLnc: linking TCGA survival data to mRNAs, miRNAs, and IncRNAs. PeerJ Computer Science. 2016;2:e67.

27. Wang J. Gene Ontology. Encyclopedia of Systems Biology. 2013;2003:797-.

28. Gaudet P, Škunca N, Hu JC, Dessimoz C. Primer on the Gene Ontology. Methods in Molecular Biology. 2017;1446:25-37.

29. Fotis C, Antoranz A, Hatziavramidis D, Sakellaropoulos T, Alexopoulos LG. Network-based technologies for early drug discovery. Drug Discov Today. 2018;23(3):626-35.

30. Boezio B, Audouze K, Ducrot P, Taboureau O. Network-based Approaches in Pharmacology. Molecular Informatics. 2017.

31. Ge Q, Chen L, Yuan Y, Liu L, Feng F, Lv P, et al. Network Pharmacology-Based Dissection of the Antidiabetic Mechanism of Lobelia chinensis. Frontiers in pharmacology. 2020;11:347.

32. Carter BZ, Mak DH, Shi Y, Fidler JM, Chen R, Ling X, et al. MRx102, a triptolide derivative, has potent antileukemic activity in vitro and in a murine model of AML. Leukemia. 2012;26(3):443-50.

33. Huang M, Zhang H, Liu T, Tian D, Gu L, Zhou M. Triptolide inhibits MDM2 and induces apoptosis in acute lymphoblastic leukemia cells through a p53-independent pathway. Molecular cancer therapeutics. 2013;12(2):184-94.

34. Fang L, Liu M, Cai L. [Hederagenin inhibits proliferation and promotes apoptosis of cervical cancer CaSki cells by blocking STAT3 pathway]. Xi Bao Yu Fen Zi Mian Yi Xue Za Zhi. 2019;35(2):140-5.

35. Krajnović T, Drača D, Kaluđerović GN, Dunđerović D, Mirkov I, Wessjohann LA, et al. The hop-derived prenylflavonoid isoxanthohumol inhibits the formation of lung metastasis in B16-F10 murine melanoma model. Food Chem Toxicol. 2019;129:257-68. 
36. Ma B, Liu X, Lu Y, Ma X, Wu X, Wang X, et al. A specific UDP-glucosyltransferase catalyzes the formation of triptophenolide glucoside from Tripterygium wilfordii Hook. f. Phytochemistry. 2019;166:112062.

37. Carter BZ, Mak PY, Wang X, Tao W, Ruvolo V, Mak D, et al. An ARC-Regulated IL1 $\beta /$ Cox-2/PGE2/ $\beta$ Catenin/ARC Circuit Controls Leukemia-Microenvironment Interactions and Confers Drug Resistance in AML. Cancer research. 2019;79(6):1165-77.

38. Wu L, Amarachintha S, Xu J, Oley F, Du W. Mesenchymal COX2-PG secretome engages NR4A-WNT signalling axis in haematopoietic progenitors to suppress anti-leukaemia immunity. British journal of haematology. 2018;183(3):445-56.

39. Han Y, Huang W, Liu J, Liu D, Cui Y, Huang R, et al. Triptolide Inhibits the AR Signaling Pathway to Suppress the Proliferation of Enzalutamide Resistant Prostate Cancer Cells. Theranostics. 2017;7(7):1914-27.

40. Zheng K, Chen Z, Sun W, Liu B, Fan D, Guo Q, et al. Hei-Gu-Teng Zhuifenghuoluo Granule Modulates IL-12 Signal Pathway to Inhibit the Inflammatory Response in Rheumatoid Arthritis. J Immunol Res. 2018;2018:8474867.

41. Wang J, Zhang Z, Li F, Chen J, Wang W. Triptolide interrupts rRNA synthesis and induces the RPL23-MDM2-p53 pathway to repress lung cancer cells. Oncology Reports. 2020.

42. Smits ELJM, Cools N, Lion E, Van Camp K, Ponsaerts P, Berneman ZN, et al. The Toll-like receptor 7/8 agonist resiquimod greatly increases the immunostimulatory capacity of human acute myeloid leukemia cells. Cancer Immunol Immunother. 2010;59(1):35-46.

43. Smits ELJM, Ponsaerts P, Van de Velde ALR, Van Driessche A, Cools N, Lenjou M, et al. Proinflammatory response of human leukemic cells to dsRNA transfection linked to activation of dendritic cells. Leukemia. 2007;21(8):1691-9.

44. Vellenga E. Constitutive NF-KB activation in AML: Causes and treatment strategies. Crit Rev Oncol Hematol. 2015;98.

45. Abdul-Aziz AM, Shafat MS, Sun Y, Marlein CR, Piddock RE, Robinson SD, et al. HIF1a drives chemokine factor pro-tumoral signaling pathways in acute myeloid leukemia. Oncogene. 2018;37(20):2676-86.

\section{Figures}




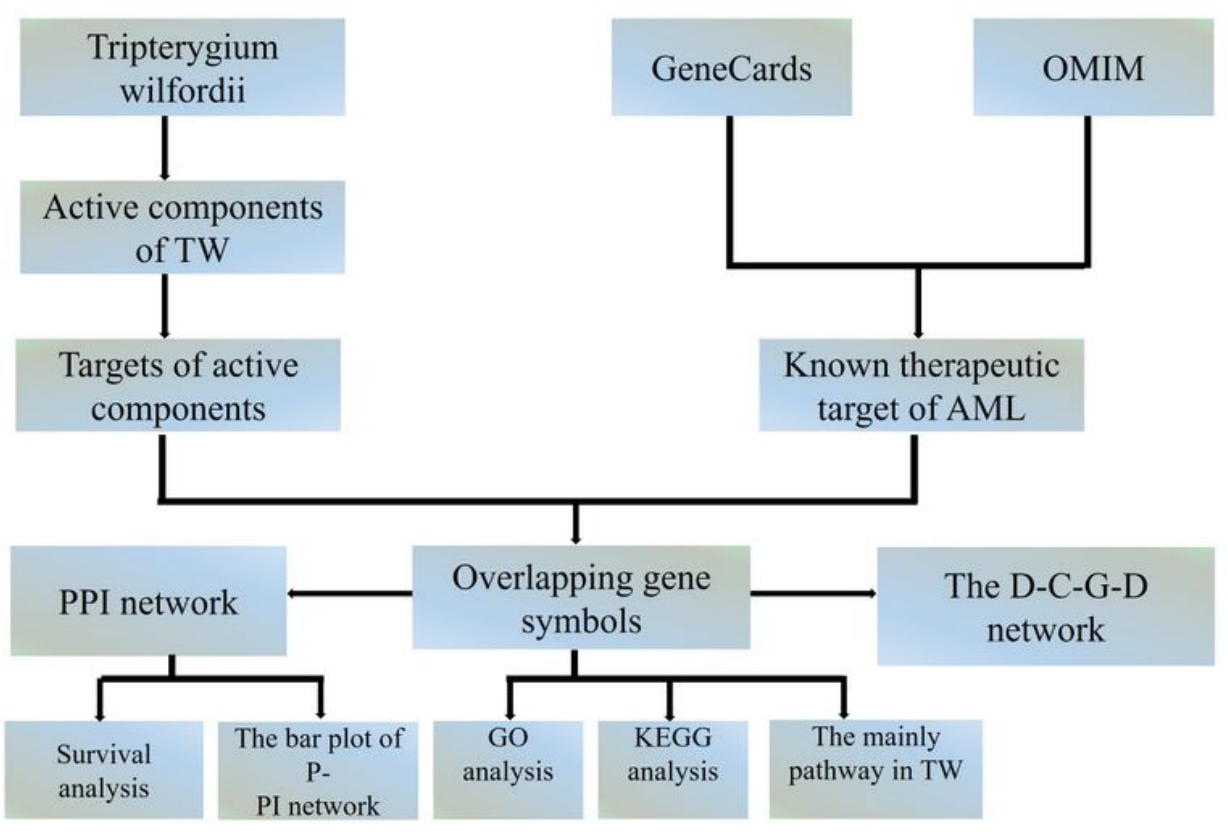

\section{Figure 1}

Flow chart of this study. TW, Tripterygium wilfordii; OMIM, Online Mendelian Inheritance in Man; GO, Gene Ontology; D-C-G-D, Drug-Compounds-Genes-Disease; PPI, protein-protein interaction. 


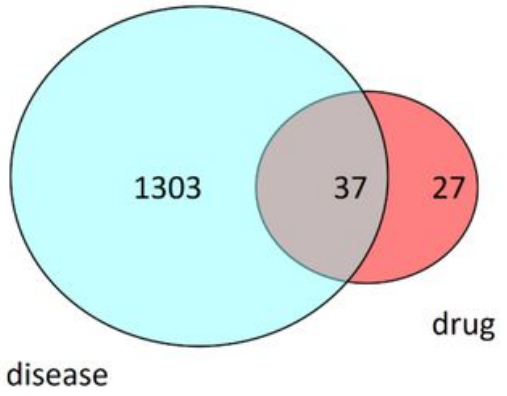

(A)

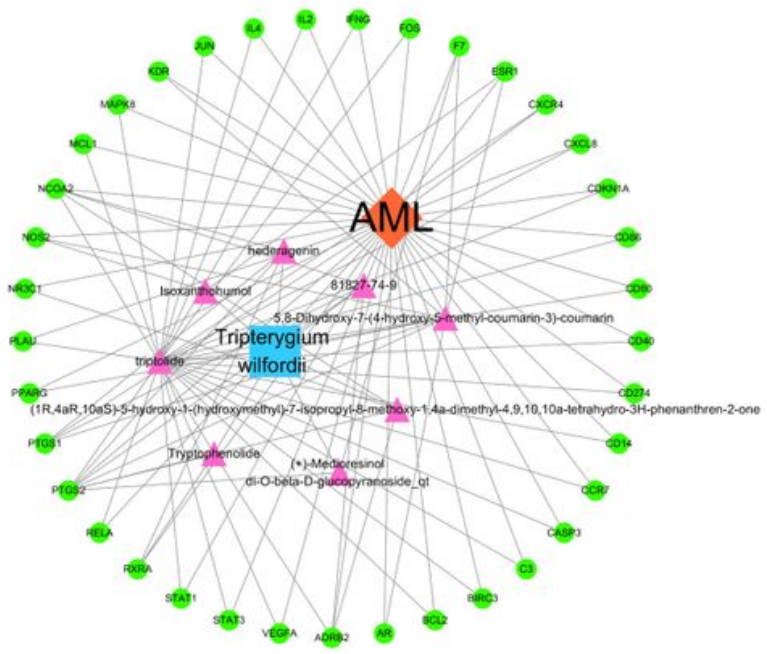

(B)

\section{Figure 2}

(A) The 37 overlapping genes between the disease and drug. (B) The Drug-Compounds-Genes-Disease network. 


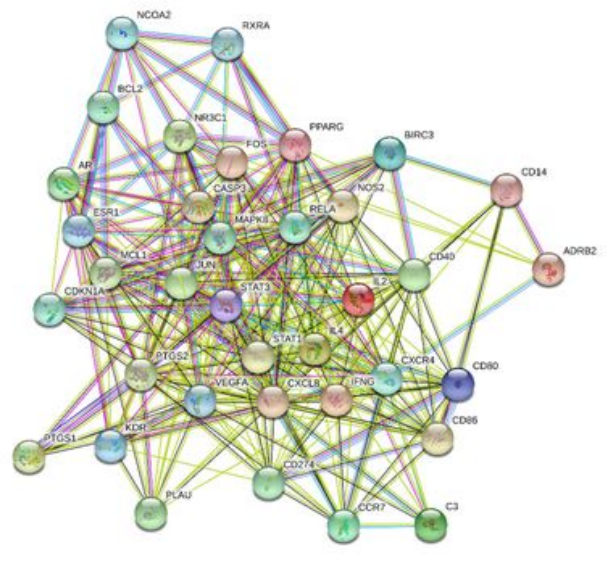

(A)

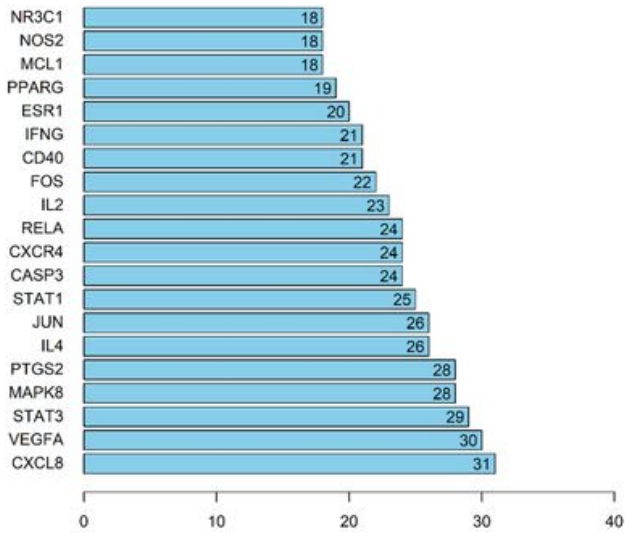

(B)

\section{Figure 3}

(A) The protein-protein interaction (PPI) network of 37 overlapping genes. (B) The bar plot of the top 20 genes in the PPI network. 

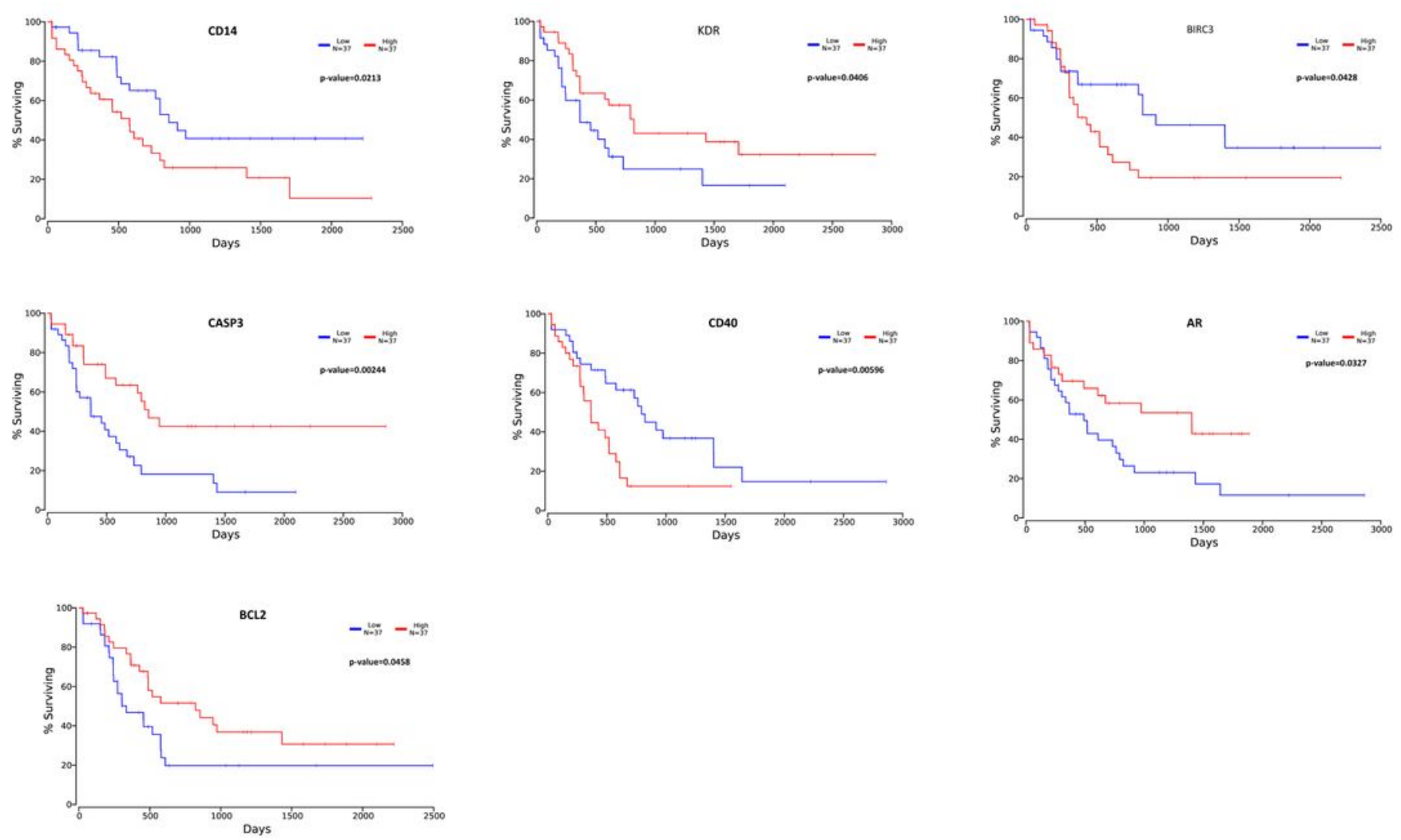

Figure 4

(A) Survival analysis of part of these target proteins. 


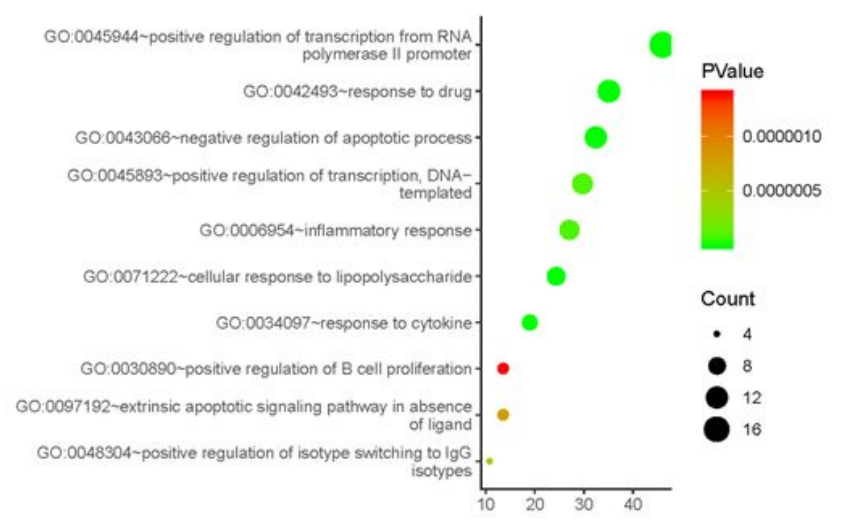

(A)

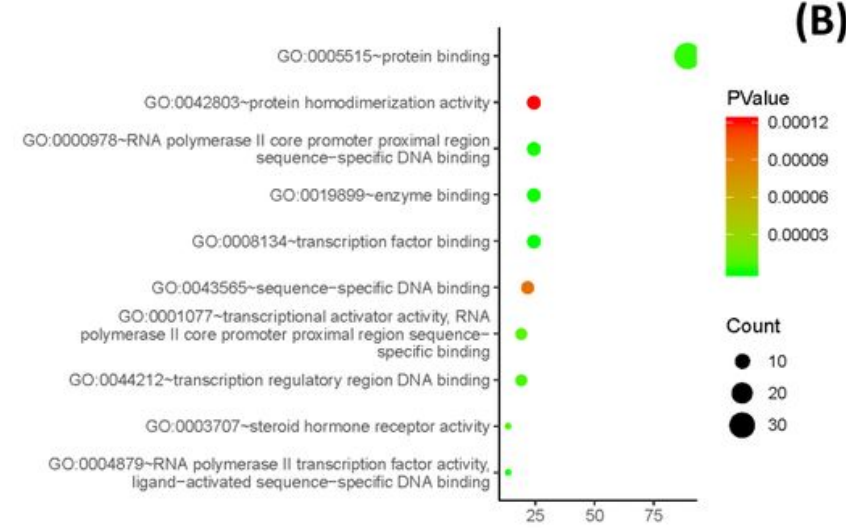

(C)

\section{Figure 5}

Gene Ontology enrichment analysis of 37 overlapping genes: (A) top 10 significantly enriched biological process; (B) top 10 significantly enriched cellular component; (C) top 10 significantly enriched molecular function. 


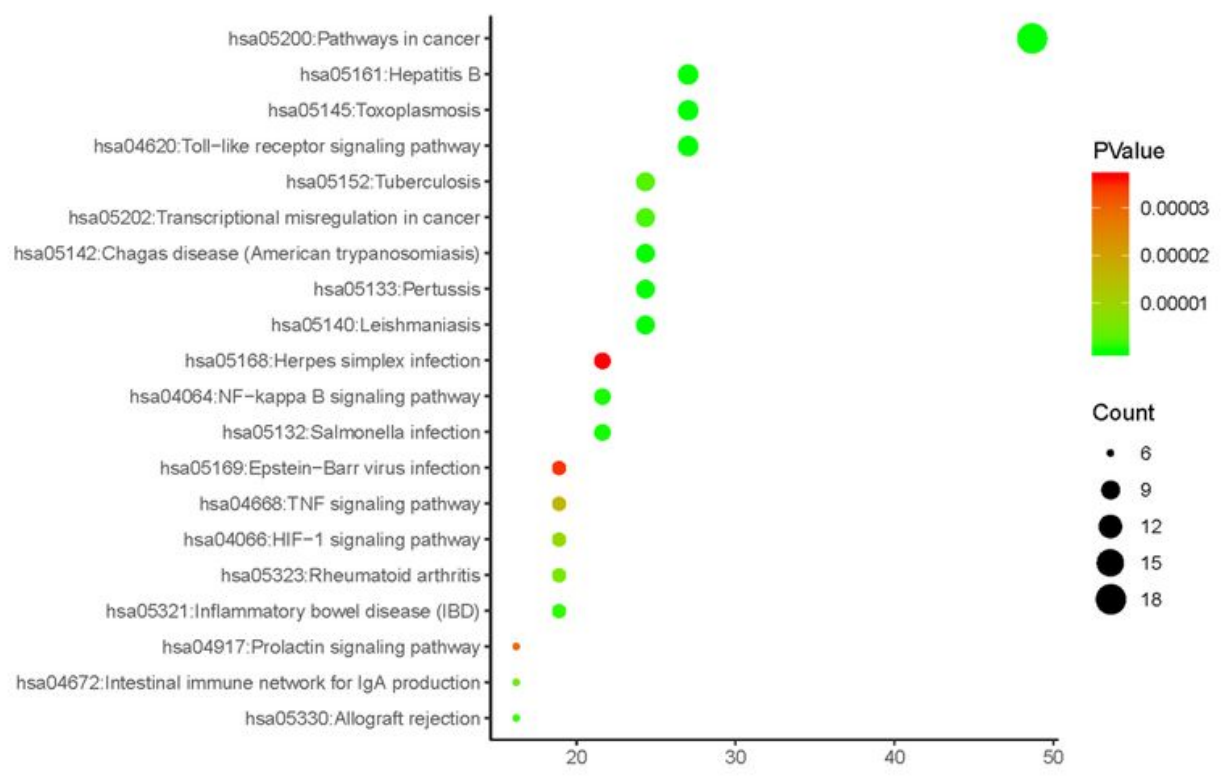

(A)

Figure 6

KEGG pathway enrichment analysis of 37 overlapping genes the top 20 significantly enriched pathways.

\section{Supplementary Files}

This is a list of supplementary files associated with this preprint. Click to download.

- SupplementaryTables2.pdf

- SupplementaryTables1.pdf 